https://doi.org/10.36592/opiniaofilosofica.v11.949

\title{
Il fenomeno della parola e del gesto in Merleau-Ponty. Per un "ritorno" al "soggetto parlante" nella Phénoménologie de la perception
}

\author{
The phenomenon of word and gesture in Merleau-Ponty. For a "return" to the \\ "speaking subject" in the Phénoménologie de la perception
}

Rosinei Aparecido Pezzini ${ }^{1}$

\section{Riassunto}

Stiamo celebrando il $75^{\mathrm{mo}}$ anniversario della pubblicazione della Phénoménologie de la perception e in questa occasione vale la pena scavare ancor di più in profondità il fenomeno della parola e del gesto in Merleau-Ponty. Il suo movimento di pensiero è un incessante richiamo al "ritorno". Potremo osservare, però, che tale "ritorno" indica un pensiero sempre incarnato e, pertanto, sempre situato. In questo articolo, ci proponiamo di gettare luce su due punti cruciali che riguardano il capitolo Le corps comme expression et la parole dell'opera di Merleau-Ponty. In un primo momento sottolineeremo il ruolo del soggetto parlante engagé nel linguaggio. Si cercherà inoltre di mostrare che, senza l'intreccio del fenomeno corpo/parola e parola/pensiero non può esistere una comunicazione vitale con il mondo. In secondo luogo verranno analizzati il fenomeno della parola in quanto vero gesto e il gesto come fenomeno d'“indicazione" nel mondo sensibile. Sulla scia di tali considerazioni, la nozione di chair del tardo Merleau-Ponty potrà essere un'éclatement di una futura teoria della chair del linguaggio.

Parole chiave: Corpo, soggetto parlante, parola, gesto, chair

\section{Abstract}

We are celebrating the 75th anniversary of the publication of the Phénoménologie de la perception and on this occasion it is worth digging even further into the depths of the phenomenon of word and gesture in Merleau-Ponty. His thought movement is an incessant call to "return". We could observe, however, that this "return" indicates an always embodied and therefore always situated thought. In this article, we aim to shed light on two crucial points concerning the chapter Le corps comme expression et la parole by Merleau-Ponty. At first we will underline the role of the speaking subject engagé in language. We will also try to show that, without the intertwining of the phenomenon body/word and word/thought, there can be no vital communication with the world. Secondly, the phenomenon of the word as a true gesture and the gesture as a phenomenon of "indication" in the sensitive world will be analyzed. In the wake of these considerations, the notion of

\footnotetext{
${ }^{1}$ Doutorando em Filosofia - Pontificia Università Gregoriana, UNIGRE, Roma/Italia. Bolsista Adveniat, ADV, Alemanha.

E-mail: rosineipessini@hotmail.com
} 
late Merleau-Ponty's chair may be an éclatement of a future chair language theory.

Keywords: Body, speaking subject, word, gesture, chair

\section{Introduzione}

In questo articolo, si cercherà di evidenziare che Merleau-Ponty è decisamente aperto a rileggere il fenomeno della parola e del gesto nel capitolo $L e$ corps comme expression et la parole della Phénoménologie. Si può, quindi, misurare la fertilità delle analisi del fenomenologo francese alla prova che ci impone, su l'invito che ci fa, d'“essere carnale" ${ }^{2}$ che siamo affatto per essere l'essere all'ascolto di una "parola parlante" 3 , di una parola che è gesto, azione e relazione. Ciò che si nota, è anzitutto, la presenza di un pensiero sensibile dell'“essere parlante" 4 , un pensiero ontologico in cammino di una vera "mutazione essenziale della parola" 5 .

Per il filosofo del soggetto parlante, la comprensione del problema del linguaggio non è solo "un problema regionale" 6 , vale a dire, di quel linguaggio già fatto, risultato di un'operazione empirica e tecnica, il linguaggio già costituito, ma è soprattutto "un tema assolutamente universale"7. È un problema cardine della filosofia. La fenomenologia del linguaggio, infatti, non è "il passaggio di un mondo confuso a un universo di significazioni chiusi" 8 , bensì il "passaggio dal mondo muto al mondo parlante"9.

Cercheremo di sviluppare in questo articolo le egide del fenomeno della parola e del ruolo del soggetto parlante. Corpo e parola: l'intreccio di questi due termini sono chiave nello sviluppo della fenomenologia del linguaggio di MerleauPonty. È possibile il darsi della chair nel cammino verso il linguaggio?

\footnotetext{
2 MERLEAU-PONTY, M., Signes, 2001, p. 254; MERLEAU-PONTY, M., Le visible et l'invisible, 1979, p. 177.

3 MERLEAU-PONTY, M., Phénoménologie de la perception, 2004, p. 238; MERLEAU-PONTY, M., Le visible et l'invisible, 1979, p. 165; MERLEAU-PONTY, M., Notes de travail inédites de 1958, 1959 et $1960, \mathrm{p}$. [342] (12).

4 MERLEAU-PONTY, M., Parcours II 1951-1961, 2000, p. 304.

5 MERLEAU-PONTY, M., Résumés de cours, 1968, p. 166.

6 MERLEAU-PONTY, M., Le visible et l'invisible, 1979, p. 165. Cf. MERLEAU-PONTY, M., Notes des cours 1958-59 et 1960-61, 1996, p. 123.

7 MERLEAU-PONTY, M., Le visible et l'invisible, 1979, p. 166.

8 MERLEAU-PONTY, M., La prose du monde, 1992, p. 25.

9 MERLEAU-PONTY, M., Le visible et l'invisible, 1979, p. 200.
} 
L'intento generale, da una parte, è di proporre una lettura degli intrecci corpo/parola e parola/pensiero nell'ottica della texture charnelle dell'assetto ontologico della chair. Dall'altra parte, invece, è di sollevare l’importanza del significato gestuale nello studio della parola. Metteremo in gioco una lettura incarnata e non naturalistica del gesto e del corpo. Ora, non ci limiteremo a fare nessuno studio del problema teorico dell'origine o della concezione generale del linguaggio, di analisi linguistica o studio semiotico del linguaggio. In fondo, la tesi che cercheremo di evidenziare è che la teoria della chair merleau-pontyana si estende in una "teoria della carne del linguaggio e della carne della storia" ${ }^{10}$.

\section{Per un "ritorno" al "soggetto parlante" engagé nel linguaggio. Il fenomeno della parola ne Le corps comme expression et la parole}

\subsection{L’intreccio di corpo e parola}

Per Merleau-Ponty, il soggetto della percezione e il soggetto parlante sono lo stesso. È il soggetto incarnato in cui il pensiero oggettivo non può più ignorare. Nella Phénoménologie, il filosofo è sensibile al tema del soggetto senzientesensibile, ma anche quello del soggetto parlante.

Nell'Avant-propos, Merleau-Ponty insiste nella necessità di "reimparare a vedere il mondo" 11. Parafrasando l'autore, potremmo dire che dobbiamo oggigiorno reimparare a parlare la parola. Reimparare a parlare la parola nel linguaggio è accogliere il vero senso del fenomeno dell'essere della parola, è acquistare uno nuovo stile d'essere nell'essere nel mondo selvaggio o grezzo, è l'aprirsi a una nuova concezione d'intercorporeità e d'intersoggettività e, infine, è il fare l'esperienza della parola nello spessore dell'essere parlante. Il fenomeno del linguaggio è il tentativo di fare un "ritorno" al soggetto parlante, al contatto tramite il corpo con il mondo aperto e intersoggettivo. L'analisi del linguaggio non è altro che il "ritorno" all'esperienza percettiva. In fondo, il soggetto parlante attraverso il suo corpo e la parola trascende verso il mondo e l'altro. Ciò che importa evidenziare è praticamente il modo in cui il soggetto parlante fa uso del suo corpo. Il corpo non è qui inteso come un "dato" definitivo e completo, ma la

\footnotetext{
10 MERLEAU-PONTY, M., Notes de travail inédites de 1958, 1959 et 196o, p. [372] (11).

11 MERLEAU-PONTY, M., Phénoménologie de la perception, 2004, p. 21.
} 


\section{4 | Opinião Filosófica, V. 11, 2020.}

sua costituzione è di per sé aperta e comunicabile. L'uso che il soggetto parlante farà del suo corpo è trascendente rispetto a quel corpo psicosomatico definito dalle scienze $^{12}$. Come si può notare, c'è un radicamento della parola in questo mondo parlante e, parallelamente, fa mostrare l'emergenza di essa nel mondo muto per rivelare l'implicazione fondamentale, il rapporto d'Ineinander, che unisce originariamente l'io e il mondo, l’io e gli altri.

Nel capitolo Le corps comme expression et la parole della Phénoménologie, si nota subito la connessione (il nexus) o l'intreccio fra questi tre termini chiave presente nel titolo stesso, cioè "corpo", "espressione" e "parola”. Si potrebbe aggiungerne altri tre che verranno sviluppati all'interno del capitolo, dato che essi si avvolgono vicendevolmente con gli altri: "pensiero", "senso" e "gesto". Com’è noto, tale capitolo evidenzia chiaramente l'impostazione del passaggio fatto da Merleau-Ponty dalla percezione al linguaggio.

Con l'intento di superare definitivamente la dicotomia classica del soggetto e dell'oggetto, il filosofo sviluppa la teoria del fenomeno della parola. In che misura il corpo rientra nell'atto d'espressione? In che modo tramite la parola e l'espressione si può riconoscere la natura ambigua del corpo? Ma che cos'è una parola? La parola è gesto? La parola è un mero segno del pensiero? Fino a che punto la parola ha influsso nel nostro modo di vivere, di parlare, di vestire nel sol primordiale che è la Terra? Tali questioni sono poste in profondità da MerleauPonty nello svolgimento del capitolo appena sottolineato della Phénoménologie. In questo testo, l'autore ci offre una prima e profonda trattazione del fenomeno della parola e del linguaggio strettamente connessi e intrecciati con il corpo.

Merleau-Ponty apre il capitolo riportando il discorso alla riscoperta del corpo. Come si può notare, nella Phénoménologie, l'autore è profondamente affezionato al problema della corporeità e del mondo. Ne Le Visible et l'Invisible, invece, soprattutto nel capitolo L'entrelacs - Le chiasme, sarà la chair l'argomento centrale del dibattito. Il filosofo sottolinea che il corpo è "una unità distinta da quella dell'oggetto scientifico"13. Dopodiché, richiama subito l'attenzione sulla necessità di una presa di coscienza riguardo a una nuova concezione del fenomeno parola. Nel trascorso del testo, il filosofo, da una parte, mette in contrasto la parola parlante incarnata venuta nel momento stesso nell'atto del parlare, cioè quella

${ }_{12}$ Cf. MERLEAU-PONTY, M., Phénoménologie de la perception, 2004, p. 230.

13 MERLEAU-PONTY, M., Phénoménologie de la perception, 2004, p. 213. 
parola autentica e, dall'altra, le parole usate nel linguaggio normale che egli definisce empirico la quale è presente "all'interno di un mondo già parlato"14. Ora, "se è parola autentica, la parola [che] fa alzare un senso nuovo" 15, è imprescindibile il richiamo di una "parola autentica".

Ma che cos'è parole authentique? Se si intende per parole authentique la verità stessa. In questo senso, uno dei meriti più grande di Merleau-Ponty sta nell'aver cercato con premura e diligenza in tutta la sua vita quella "verità ultima"16. Infatti, ne Le Visible et l'Invisible, l'autore fa notare che "non siamo noi a parlare, è la verità a parlarsi in fondo alla parola" ${ }^{17}$. Tramite l'analisi dettagliata della parola e dell'espressione, Merleau-Ponty propone alla fine del capitolo alcune delle considerazioni importanti. In un primo momento, la necessità di "riconoscere la natura enigmatica del corpo proprio e [che] l'esperienza del corpo proprio ci rivela un modo d'esistenza ambiguo"18. Il rovescio del corpo è la sua ambiguità. Il suo potere è quindi essere ambiguità nel suo proprio modo d'essere. Nel cuore di questo movimento d'esistenza ambiguo, Merleau-Ponty parla di un

14 MERLEAU-PONTY, M., Phénoménologie de la perception, 2004, p. 224. Riguardo al tema del linguaggio empirico, Fanizza sintetizza "il linguaggio empirico è quello che distingue fra pensiero e parola, che creda a due distinte vie del pensiero e della parola, che tratta quest'ultima come uno strumento secondario da usarsi dopo che il pensiero è stato già formulato" (FANIZZA, F., Motivi estetici nella fenomenologia di Merleau-Ponty. Aut Aut, 1961, p. 527).

15 MERLEAU-PONTY, M., Phénoménologie de la perception, 2004, p. 236. (Corsivi miei).

16 MERLEAU-PONTY, M., Le visible et l'invisible, 1979, p. 201. Nella Phénoménologie già si costata un Merleau-Ponty affascinato per la ricerca della verità, per esempio, come si veda: "ogni verità di fatto è verità di ragione, ogni verità di ragione è verità di fatto" (MERLEAU-PONTY, M., Phénoménologie de la perception, 2004, p. 454). "L'essere-alla-verità non è distinto dall'essere al mondo" (MERLEAU-PONTY, M., Phénoménologie de la perception, 2004, p. 455). Al riguardo, in una nota interna a uno dei saggi raccolti in Sens et non-sense, La metaphysique dans l'homme, scritto nel'47, Merleau-Ponty faceva esplicito riferimento alla necessità di "descrivere precisamente il passaggio alla fede percettiva alla verità esplicita quale la si incontra al livello del linguaggio. [...] Contiamo di farlo in un lavoro consacrato all'Origine della verità" (MERLEAU-PONTY, M., Sens et non-sens, 1948, p. 167). A questo riguardo, si veda il commento di Noble: "dans la Phénoménologie, le langage est lié à la question du sens, et surtout à celle de l'émergence du sens dans notre expérience perceptive. Mais dans cette note, nous apercevons un autre aspect de ce thème, une autre zone d'ombre: qu'en est-il précisément de la question de la vérité?" (NOBLE, S. A., "Entre le silence des choses et la parole philosophique". Chiasmi International, 2005, p. 130131). Ed ancora nel testo del ' 52 , col quale Merleau-Ponty presenta la sua candidatura al Collège de France, poi pubblicato nel '62 nella Reveu de Métaphysique et de Morale col titolo Un inédit de Maurice Merleau-Ponty, il filosofo accenna il titolo a uno dei suoi importanti progetti. Egli si esprime così: "en attendant de traiter complètement ce problème dans l'ouvrage que nous préparons sur l'Origine de la Vérité" (MERLEAU-PONTY, M., Un inédit de Merleau-Ponty. Revue de Métaphysique et de Morale, 1962, p. 406). L'opera incompiuta Le Visible et l'Invisible è il titolo probabilmente definitivo che avrebbe preso il posto di l'Origine de la Vérité. Per una trattazione del tema della verità, si rimanda al WALDENFELS, B., Vérité à faire. In: BOETTI, A.-M. S., La prosa del mondo, 1990, p. 81-90.

17 MERLEAU-PONTY, M., Le visible et l'invisible, 1979, p. 236.

18 MERLEAU-PONTY, M., Phénoménologie de la perception, 2004, p. 239-240. 


\section{6 | Opinião Filosófica, V. 11, 2020.}

potere di "una specie di sfuggimento" 19 che getta l'essere umano verso l'acquisizione di nuove modalità di comportamenti e di nuovi gesti. Questo sfuggimento costante abita l'uomo, ma il suo sfuggire non rompe il suo engagement ontologico, anzi lo fa diventare più engagé di ciò che era. Anche il linguaggio è di per sé ambiguo e obliquo. Nel secondo momento, egli definisce che il corpo "non è più un aggregato di particelle [...] o anche un intreccio di processi definiti una volta per tutti; esso non è dove è, non è ciò che è” ${ }^{20}$. In una terza tappa, il filosofo ammette un'altra sua teoria sviluppata all'interno del capitolo, cioè che "il gesto o la parola trasfigurano il corpo"21. Alla fine dei conti, conclude affermando che "è il corpo che mostra, è il corpo che parla. [...] Sia che si tratti del corpo dell'altro o del mio proprio corpo, ho un solo modo di conoscere il corpo umano, quello di viverlo. [...] Io sono dunque il mio corpo"22. Il corpo quindi non è più un oggetto, ma è il corpo trasfigurato tramite il gesto e la parola, è un "corpo che parla". Oltre un corpo che pensa, è un corpo che parla, un corpo che vive, un corpo gestuale, un corpo engagé. È a partire da questa cornice teorica che arriviamo quindi alla questione che più ci interessa, e cioè il ruolo del corpo nel linguaggio. Il "corpo che parla" è uno dei modi in cui si manifesta l'azione umana nella comunità vivente. Infatti, è il modo di vivere ed agire per eccellenza in quanto corporeità. $\mathrm{Al}$ di là di tutto, nella filosofia merleau-pontyana, il problema del corpo e del mondo consiste in ciò, "che tutto vi rimane"23.

Ciò che comanda la concezione merleau-pontyana del linguaggio nella Phénoménologie è precisamente l'attenzione rivolta alla descrizione del "fenomeno della parola e l'atto espresso di significazione" 24 . La parola è un fenomeno originario. Ciò che è qui messo in gioco è quel tentativo di un "ritorno" alla parola stessa. Con l'espressione "ritorno" alla parola, Merleau-Ponty vuole restituire ad essa il sorgere della sua esperienza originaria dove si costituisce il mondo parlante reale e che la verità appare come un'éclatement. Il "ritorno" alla parola non è altro che l'intento di svelare il senso che ha la parola sullo sfondo del silenzio primordiale che la precede e non cessa di accompagnarla; oltre questo, è anche il fare apparire dal silenzio la nudità di sua chair. La linea guida proposta da

19 MERLEAU-PONTY, M., Phénoménologie de la perception, 2004, p. 230.

20 MERLEAU-PONTY, M., Phénoménologie de la perception, 2004, p. 239.

${ }^{21}$ MERLEAU-PONTY, M., Phénoménologie de la perception, 2004, p. 239. (Corsivi miei).

22 MERLEAU-PONTY, M., Phénoménologie de la perception, 2004, p. 239; 240. (Corsivi miei).

23 MERLEAU-PONTY, M., Phénoménologie de la perception, 2004, p. 240.

24 MERLEAU-PONTY, M., Phénoménologie de la perception, 2004, p. 213. 
Merleau-Ponty è comprendere il linguaggio nella prospettiva del fenomeno della parola e non della lingua, come proponeva Saussure nel Corso di linguistica generale $^{25}$. Per Merleau-Ponty, Saussure si astiene dal pensare l'interazione del fenomeno del mondo sensibile e della corporeità nello studio della lingua. Il filosofo francese, invece, cerca di chiarire il mondo fenomenico come base dell'apparizione della lingua. Se la lingua non fosse incarnata in un corpo, si potrebbe dire che esiste una lingua? In ogni caso, esaminare la linguistica saussuriana non è il nostro obiettivo in questa sede. Prendendo su serio il problema della parola, il Nostro prende cura della possibilità di far risaltare entrambi gli aspetti.

Merleau-Ponty postula l'esistenza di una parola parlante e di una parola parlata. L'articolazione e l'implicazione reciproca della parola parlante e della parola parlata rivelano le facce dello stesso oggetto e costituiscono il proprio del linguaggio rispetto a tutte le altre forme d'espressione. $\grave{E}$ alla luce di tale concezione che il filosofo distingue il gesto originario espressivo "allo stato nascente" 26 dal soggetto corporeo come parola parlante da quello logico-discorsivo già acquisito della parola parlata. Ma la fenomenologia è un vero tentativo di cogliere la parola parlante come linguaggio nel suo stato nascente. La parola parlante non è altro che il gesto inaugurale ed esistenziale fondamentale di dar nome al mondo e di significarlo: "nominare l'oggetto significa farlo esistere o modificarlo: Dio crea gli esseri nominandoli e la magia agisce su questi parlando di essi" 27 . La parola parlante in quanto atto corporale produce l'istituzione verbale o culturale. Questa istituzione è la parola parlata o lingua in quanto sistema convenzionale.

Ciononostante, distinguendo tra parola parlante e la parola parlata, Merleau-Ponty non vuole stabilire una gerarchia metafisica tra una parola autentica ed una parola inautentica ${ }^{28}$. È anche vero che il filosofo non vuole nemmeno squalificare la parola parlata come derivazione secondaria. In altri termini, è all'interno di questo intreccio, cioè tra la parola parlante e la parola parlata che l'operazione espressiva e l'intenzione di significazione si rivelano a se

25 Cf. SAUSSURE, F., Corso di linguistica generale, p. 31.

26 MERLEAU-PONTY, M., Phénoménologie de la perception, 2004, p. 238.

27 MERLEAU-PONTY, M., Phénoménologie de la perception, 2004, p. 217.

28 Secondo Thierry, questa distinzione tracciata tra parola parlante e parola parlata permette di "mettere in evidenza una connessione fra i due. In realtà non ci sono due tipi di parole, ma lo stesso fenomeno del linguaggio" (THIERRY, Y., Du corps parlant, 1987, p. 39). 


\section{8 | Opinião Filosófica, V. 11, 2020.}

stessi come capace di incarnarsi come cultura. In fondo, si potrebbe dire che la parola parlante avviene nel momento stesso dell'atto del parlare, nel momento in cui il suo senso e la sua significazione sta per incarnarsi e la parola parlata è già l'atto incarnato compiuto. La parola parlante scivola (glisser) su lo strato sedimentario della parola parlata. Ora, esse sono come due assi incrociati con cui le nostre espressioni s'éclatent verso il mondo e si comunicano ad altri. Attraverso "un solo éclatement d'Essere" 29 siamo in grado d'uscire, pur rimanendo in noi stessi con l'intento di parlare dall'interno dell'essere. In questo nuovo assetto ontologico, il parlare non è soltanto pronunciare delle parole o dei suoni, ma è far éclater un modo di vivere l'esistenza dal cuore dell'Essere, "facendomi mondo e facendole carne"30. In altri termini, parlare è éclater verso l'altro.

Torniamo dunque al problema del corpo. Come abbiamo menzionato prima, il corpo divenne un argomento fondamentale in tutta la trattativa del problema della parola. Per Merleau-Ponty è impossibile trattare della problematica della parola senza toccare in profondità il tema della corporeità. Il corpo e la parola si costituiscono quindi simultaneamente. Carne del corpo e carne della parola, dato che ogni parola è intessuta di carnalità e di gestualità. Questa considerazione non esiste normalmente nei linguisti. Si tratta, infatti, del connettersi di corpo e linguaggio, quell'empiétement dell'uno sull'altro. L'interrogazione ontologica di tale connettersi sarà infine concepita ne Le Visible et l'Invisible come chiasmo di corpo e linguaggio. Si può dedurre fin d'ora, in tutta coerenza, che c'è nella Phénoménologie, rispetto la presenza di un'esperienza corporea, una trattazione del corpo sviluppata attraverso una teoria fenomenologica dell'engagement.

L'engager in Merleau-Ponty è un concetto chiave per comprendere l'autenticità di una presenza impegnata del nostro corpo nelle strutture tipiche di un mondo. Tale termine appare parecchie volte nel testo della Phénoménologie. Il corpo s'engage. Detto in altri termini, l'essere corpo è, quindi, essere engagé. Ma che cosa vuol dire? Si tratta di costruire la realtà del corpo come un essere che è ciò che è, cioè engagé al mondo selvaggio e muto. In fondo, il corpo diventa ciò che sempre era: engagé, quindi, mai désengagé. A questo punto, bisogna dar ragione a Merleau-Ponty: noi siamo engagés. La realtà ontologica

\footnotetext{
29 MERLEAU-PONTY, M., Le visible et l'invisible, 1979, p. 313. (Corsivi miei).
}

30 MERLEAU-PONTY, M., Le visible et l'invisible, 1979, p. 176. 
dell'engagement del soggetto al mondo è grazie alla sua chair e la chair du monde. "Io m'engage con il mio corpo fra le cose, esse coesistono con me come soggetto incarnato" ${ }^{31}$. Alla luce di questa citazione si può, allora, comprendere cosa vuol dire s'engager: impegnarsi, ingaggiarsi, lanciarsi. Non siamo soltanto un essereal-mondo, ma un essere ingaggiato ad esso. Il nostro impegno (engagement) nel mondo è effettivo, non superficiale. Non siamo soggetti acosmici, ma soggetti impegnati nella lotta comune. Nel mondo linguistico il soggetto è già impegnato nella parola. Apparentemente questi esempi sembrano essere sufficienti, ma alla fine della frase Merleau-Ponty ritorna ed accenna il personaggio principale del fenomeno dell'engagement: il soggetto incarnato ${ }^{2}$.

In linea con questa considerazione, s'engager vuol dire anche "incarnarsi". L'incarnarsi è l'apertura alla coesistenza. Abito con gli altri uomini come i soggetti incarnati che hanno un corpo simile al mio corpo. Allo stesso tempo, abitiamo in mezzo alle cose. Nell'opera del '61, L'Eil et l'Esprit, l'autore scrive su lo stesso argomento: "le cose e il mio corpo sono fatti della stessa étoffe" 33 . È certo che il mio corpo e i corpi degli altri sono fatti anche della stessa étoffe e, quindi, entrambi sono chairs du corps. Il punto è che il progetto merleau-pontyano dell'intreccio corpo e parola si inscrive, in modo più generale, entro un atteggiamento di pensiero che va di quel "modo d'esistenza ambigua" 34 del corpo al chiasmo, dalla filosofia dell'incarnazione alla ontologia della chair dunque, dalla Phénoménologie all'opera Le Visible et l'Invisible.

\subsection{Parola, espressione e senso}

Di per sé, il corpo stesso è già espressione incarnata per eccellenza. È attraverso lo studio sul linguaggio che sorge in tutta la sua grandezza la nozione centrale della filosofia dell'espressione. Anzitutto, "espressione" è movimento carnale. La comunicazione dei miei pensieri non è altro che movimento, giacché attraverso di esso mi volgo verso qualcuno. Ed ogni movimento di questo "versarsi" è carnale. Oltre questo, l'atto di esprimere è il modo con cui si manifesta lo stato d'animo o delle emozioni, è il far apparire. L'uomo si esprime in quanto

${ }_{31}$ MERLEAU-PONTY, M., Phénoménologie de la perception, 2004, p. 226. (Corsivo mio).

$3^{2}$ Cf. MERLEAU-PONTY, M., Phénoménologie de la perception, 2004, p. 79; 112; 191; 226; 235; $239 ; 245 ; 246 ; 258 ; 376 ; 504 ; 515$.

33 MERLEAU-PONTY, M., L'œil et l'esprit, 2007, p. 21. (Corsivo mio).

34 MERLEAU-PONTY, M., Phénoménologie de la perception, 2004, p. 240. 
corpo e in quanto soggetto parlante. Siamo, quindi, nel campo fenomenologico del "ritorno" per eccellenza. Soltanto a titolo di illustrazione, possiamo rilevare il fenomeno dell'espressione presente nelle arti, ad esempio, La montagna SainteVictoire di Cézanne. Esso ha un potere di espressività che va oltre; la manifestazione dell'espresso è al di là dall'espresso stesso. Scriverà, infatti, ne $L a$ Prose du Monde che "l'espressione non è mai completamente espressione, l'espresso non è mai del tutto espresso"35. L'espressione non è prima nel senso espressivo e l'espresso non è semplicemente derivato dall'espressione, poiché l'espressione si manifesta proprio nell'espresso. In altri termini, si potrebbe dire che "l'espressione si dissolve di fronte all'espresso" 36 ed è in esso attraverso un altro modo d'essere. È quindi il sol primordiale del potere espressivo in quanto fenomeno, di quella "produttività che costituisce l'essenza più profonda dell'uomo" ${ }^{37}$. Se è vero che l'essenza della parola è la carnalità, lo sforzo di Merleau-Ponty si trasforma in una sorta di ricominciamento o dell'altro inizio della storia esistenziale della parola stessa. Affrontare questa impresa è scavare il rapporto tra la parola e la chair.

Da questo punto di vista, che cosa esprime il linguaggio? Il linguaggio esprime dei pensieri come il desiderio con la carezza? In fondo, il linguaggio ha il potere di far nascere l'espresso. Il termine "espressione" è sinonimo di visibilità, visto che ogni visibilità è per eccellenza l'atto di espressività. In più, se l'espressività stessa è l'atto dell'apparire, quindi, l'autore ci porta al cuore della fenomenologia. La parola chiave che apre il cosiddetto capitolo è che il corpo ha "un potere di significazione" 38 e è il luogo d'espressione per eccellenza. MerleauPonty propone un punto di vista alternativo alle proposte delle tradizioni cartesiane e dello psicologismo e ci invita a riflettere sul fatto che il corpo si trova almeno collegato a tre dimensioni: la parola, il gesto e persino, oltre, il silenzio all'interno del quale sorge per costituirsi come una nuova espressione. MerleauPonty si affida alla parola per comprendere il corpo in quanto luogo d'espressione. Il ricupero del corpo espressivo si articola su una filosofia del linguaggio. La parola è riconosciuta quale esempio privilegiato dell'essenza dell'espressione. La parola è solo un semplice concetto verbale? È un'operazione intellettuale?

35 MERLEAU-PONTY, M., La prose du monde, 1992, p. 52.
36 MERLEAU-PONTY, M., Phénoménologie de la perception, 2004, p. 462.
37 MERLEAU-PONTY, M., Phénoménologie de la perception, 2004, p. 238.
38 MERLEAU-PONTY, M., Phénoménologie de la perception, 2004, p. 213.

Revista Opinião Filosófica - ISSN: 2178-1176 - Editora Fundação Fênix. www.fundarfenix.com.br 
L'uomo può parlare così come il poeta può poetare, lo scrittore può scrivere, il pittore può dipingere e il tenore può cantare. Merleau-Ponty dice con lungimiranza alla fine dell'Éloge de la philosophie che l'uomo è colui "che si sveglia e parla" 39 . L'uomo parla, egli è homo loquens. L'uomo può risvegliarsi dal sonno dogmatico, idealistico ed intellettualistico. La parola è il filo carnale che unisce ed intreccia i soggetti parlanti: linguaggio parlato, linguaggio scritto, linguaggio simbolico e linguaggio cantato. In realtà, davanti al fenomeno della parola, non si rileva il patrimonio di parole usate per ciascuno di essi, ma il modo di farne uso. Allora, la "parola" lascia di essere intesa solo dal punto di vista delle leggi della meccanica nervosa, degli stimoli o degli stati di coscienza per "prendere posto nel circuito di fenomeni” 40 . È grazie a questo circuito di fenomeni che il problema del linguaggio riesce ad uscire dalle concezioni delle psicologie empiristiche e quelle intellettualistiche. Ora, queste due concezioni, anche se dal punto di vista metodologico sono opposte, riguardo la "parola" hanno qualcosa in comune, perché per entrambe la parola "non ha significato. [...] Essa è ancora priva di efficacia propria" ${ }^{41}$. Per quanto concerne la spiegazione dell'apparizione della parola, l'empirismo e l'intellettualismo falliscono nella loro impresa: "nella prima concezione, siamo al di qua della parola come significativa; nella seconda, al di là; nella prima, non c'è nessuno che parla; nella seconda c'è sì un soggetto, ma questo non è il soggetto parlante, è il soggetto pensante" ${ }^{2}$. Nell'atteggiamento empirico la parola è soltanto una semplice reazione meccanica dell'organismo, invece nell'intellettualismo la parola e il senso sono separati l'uno dall'altra. La "parola" non è una busta o scatola vuota e inerte. Per sfuggire da tali concezioni, Merleau-Ponty postula una nuova idea di parola, cioè che "la parola ha un senso" 43 .

La parola ha, quindi, un senso. La parola precede il senso; ecco perché non abbiamo esaurito il senso della parola tramite lo studio del loro unico significato concettuale. Purtroppo il mondo ha perso il senso del senso della parola e, perciò, l'ha svuotata e l'ha screditata. Bisogna tornare a prendersi cura delle parole, del loro significato che rende riconoscibile la verità. Se la parola si corrompe, l'uomo si perde. La perdita del contatto con la realtà è la perdita del senso che è la verità

39 MERLEAU-PONTY, M., Éloge de la philosophie, 1960, p. 63.

40 MERLEAU-PONTY, M., Phénoménologie de la perception, 2004, p. 214.

41 MERLEAU-PONTY, M., Phénoménologie de la perception, 2004, p. 216.

42 MERLEAU-PONTY, M., Phénoménologie de la perception, 2004, p. 216.

43 MERLEAU-PONTY, M., Phénoménologie de la perception, 2004, p. 216. 
stessa. Una simile descrizione come, per esempio, la solita frase: "parola senza senso", quale che sia questa analisi, potrebbe forse formularsi così: "la parola ha un senso, e, al di fuori di essa, senza senso". In realtà, questo "senza" del senso non è la stessa cosa del "non-senso". Guardando oltre, forse vuol dire che il senso o il significato si è ritirato dalla parola, "ma visibilmente la parola quando perde il suo senso si modifica perfino nel suo aspetto sensibile, si svuota" 44 . Nella misura in cui la parola si svuota e si degrada, la menzogna fa da padrona alla parola stessa. Allora, essa perde il suo rapporto con il reale, diventa pura astrazione, è la rottura o la corruzione del carattere comunicativo. La menzogna è l'adulterazione della parola. Merleau-Ponty vuole restituire la vera fisionomia alla parola: la parola "è", ecco l'unica maniera di definire il suo modo d'essere. Tutelare la parola è tutelare la verità. Siccome ci troviamo immersi in una filosofia del lien oppure dell'entrelac, si può ammettere che c'è qui un legame (lien) stretto fra la parola e la verità, la parola e il suo senso come fra il mondo linguistico e il corpo proprio. Il tema del lien è molto apprezzato dal Nostro. Si può riassumere affermando che "il legame della parola al suo senso vivente non è un legame esteriore d'associazione" 45 .

"La parola ha un senso". Il senso è essenzialmente inerente alla parola; la parola come l'espressione e il senso come l'espresso sono inseparabili. Quando Merleau-Ponty scrive nell'Avant-propos che "siamo condannati al senso" 46 sembra indicare che non possiamo uscire dall'universo dal senso neanche dal linguaggio, perché siamo al mondo, engagé in esso. Quell'essere parola della parola parlante venuta al mondo parlante è quindi il fenomeno dell'incarnazione. Da questo punto di vista, la parola non è più un mero simbolo, un suono o un segno esteriore come l'ammetteva la concezione intellettualista ed empirista. Il senso dell'essere della parola, in quanto si svela al mondo muto e lo fa diventa parlante, costituisce il fenomeno dell'essere della parola. Questo "senso" ha anche esso un essere, in base al quale si rivela, si manifesta, si espressa ed appare tramite l'essere della parola in quanto l'espressione. Perché la parola abbia "senso" e perché si possa di conseguenza interrogarci sulla parola, bisogna che il non-senso sia dato in qualche modo. Siamo qui nel piano dell'essere, non innanzi alla conoscenza linguistica.

\footnotetext{
44 MERLEAU-PONTY, M., Phénoménologie de la perception, 2004, p. 234.

45 MERLEAU-PONTY, M., Phénoménologie de la perception, 2004, p. 234. (Corsivi miei).

46 MERLEAU-PONTY, M., Phénoménologie de la perception, 2004, p. 20.
} 
Tuttavia il senso è sempre là, intessuto di carnalità. Barbaras sostiene che "il senso è solo una 'piega' nel tessuto della parola" 47.

Soltanto a titolo di chiarimento, ci aiuterà a capire meglio evidenziando un esempio riguardo al senso o al significato che ha la parola. La "sveglia", per esempio, è una parola che porta il senso. Sentire lo squillo della sveglia come richiamo, vuol dire alzarsi. La parola "sveglia" ha in sé il senso, cioè quello di svegliarmi. Da questo punto di vista, cogliere il senso della suoneria significa alzarmi al suo richiamo e così da questo evento si nota come la parola "sveglia" svela il suo senso, cioè quello di svegliare. Ecco, dunque, la sua carnalità. Per il filosofo, la parola, lungi d'essere un semplice segno, abita gli oggetti. Il senso non si svincola dalla parola, è appartenente e s'ancre ad essa. In ogni parola espressa, il senso trasmesso all'ascoltatore eccede il proprio linguaggio e il pensiero già costituito. L'uomo è, dunque, senso e, allo stesso tempo, fa apparire ed esprime il senso.

\subsection{Parola e pensiero come «avvolti l'uno nell'altro»}

Sembra che ci troviamo davanti a questo nuovo problema dibattuto da Merleau-Ponty nello stesso capitolo della Phénoménologie: il rapporto fra parola e pensiero. La parola è un accessorio esteriore del pensiero? È condizionata da esso? Soggetto parlante e soggetto pensante. Come pensare tale groviglio senza cadere di solito in tali cosiddette concezioni classiche dell'intellettualismo e dell'empirismo? Si dice, di solito, così nel dare un consiglio a qualcuno: "pensa prima di parlare”. $\mathrm{E}$ vero che è una condotta che molti seguono e che impegna la capacità intellettiva per respingere ogni sorta di fraintendimento riguardo ciò di cui si parla. Quante volte abbiamo sentito dalle persone tale suggerimento con l'intento di prevenirci da qualsiasi sbaglio o equivoco suscitato dall'aver male usato le parole, che è spesso causa di risentimenti, litigi o malinteso? Così, dopo un avvenimento determinato da litigio nei confronti della fidanzata, il suo amico gli dice: "se avessi pensato prima, non avresti litigato". Questa osservazione ci consente di mettere al

\footnotetext{
47 BARBARAS, R., Le tournant de l'expérience, 2013, p. 193. La piega (pli) pensata da MerleauPonty è quello spazio onto-topologico della differenza tra dicibile e indicibile, quell'articolazione dell'apertura nell'unità in divenire propria dell'intreccio di corpo e parola. Per quanto riguarda la nozione di piega e la rilevanza ontologica che riveste all'interno della filosofia dell'ultimo MerleauPonty, si rimanda a GAMBAZZI, P., La piega e il pensiero. Aut Aut, 1994, p. 21-47; LAWLOR, L., Le chiasme et le pli. In: CARIOU, M. (org). Merleau-Ponty aux frontières de linvisible, 2003, p. 191203; SEGGIARO, N., La chair et le pli, 2009, p. 23-51.
} 
primo posto "il pensare", per dopo venire "il parlare". Pensiero e parola. C'è infatti dietro a questa posizione un'inclinazione verso l'intellettualismo. In questo caso, la parola diventa il segno del pensiero, l'immagine verbale di esso o la maniera di designare l'oggetto. Ci si potrebbe domandare: il pensiero viene prima della parola o la parola viene prima del pensiero? Cosa ne pensa Merleau-Ponty? Uno dei compiti dei filosofi, soprattutto nel caso della parola, l'operazione espressiva per eccellenza, è che "si può parlare sulla parola" 48 . Certo l'uomo può parlare e parlare del suo parlare, vale a dire che il fenomeno della parola offre la possibilità di ripiegare su se stessa, cioè di ritornare su di sé.

Se vogliamo penetrare più a fondo la questione "parola e pensiero", dobbiamo domandarci subito: la parola presuppone il pensiero? Il pensiero è fondamento della parola? Dobbiamo tener presente che Merleau-Ponty cerca almeno di restituire all'atto del parlare la sua vera fenomenalità. Di per sé, l'essenza dell'atto del parlare rivela la condizione della parola in quanto fenomeno: il suo modo d'essere è essere un essere che mi svela, che mi appare in quanto essere parlante. Il parlare, secondo lui, non è un unirsi all'oggetto attraverso un'intenzione di conoscenza o una rappresentazione, ma è un accedere al mondo selvaggio e muto da parte di un soggetto incarnato. La parola "è l'eccesso della nostra esistenza sull'essere" 49 del mondo parlante. Esso non è quel mondo instituito e parlato dove fruisce soltanto la parola parlata come se fosse un patrimonio già acquisito. Resta inteso che tale "eccesso", vuol dire che la parola è sempre abbondante, il senso del suo essere è andare oltre (dépassement) i suoi soliti limiti. La parola non è più considerata come un fatto interno alla struttura della lingua, come un risultato e, perciò è una parola sempre parlata ma non parlante. Così, la parola in quanto dépassement richiama qui alla "pienezza dell'essere" 50 , il motivo per cui si svela sempre la prima parola quando si tratta di parola parlante della nostra esistenza sull'essere. Questo evento si dà nella rottura dal silenzio che "come un'onda, si raccoglie e si riprende per proiettarsi al di là di se stessa" ${ }^{1}$.

Per questo, l'intento del filosofo è di evidenziare la vera fenomenalità dell'apparire dell'atto del parlare. L'intenzione di parlare si trova, dunque, "in

48 MERLEAU-PONTY, M., Phénoménologie de la perception, 2004, p. 231.
49 MERLEAU-PONTY, M., Phénoménologie de la perception, 2004, p. 239.
50 MERLEAU-PONTY, M., Phénoménologie de la perception, 2004, p. 239.
51 MERLEAU-PONTY, M., Phénoménologie de la perception, 2004, p. 239.

Revista Opinião Filosófica - ISSN: 2178-1176 - Editora Fundação Fênix. www.fundarfenix.com.br 
un'esperienza aperta, essa appare, come l'ebollizione in un liquido, quando, nello spessore dell'essere, zone di vuoto si costituiscono e si spostano verso l'esterno" ${ }^{2}$. Da tale punto di vista, l'uomo è l'unico essere che può compiere l'atto dell'apparire della parola tramite il parlare. L'uomo, quindi, s'engage mentre parla. È vero che, la parola, in colui che parla, cioè il soggetto parlante, non traduce semplicemente un pensiero già fatto o istituito, ma lo compie nell'atto stesso del parlare. Oltre a questo, si potrebbe ammettere che colui che ascolta accoglie il pensiero della parola stessa. Ora, che cosa la parola udita gli porta? Anzitutto, c'è un'operazione reciproca tra uno e l'altro. Se l'ascoltatore accoglie la parola di colui che parla e la capisce, ciò vuol dire che quella parola non è "un contributo estraneo" 53 a lui, ma che nel suo campo linguistico, l'aveva già bene compresa, altrimenti non esisterebbe comunicazione. In verità, di fronte al soggetto parlante e l'ascoltatore c'è un linguaggio comune perché appartengono allo stesso mondo linguistico. La comprensione della parola udita proviene nel momento in cui ci engageons nel modo di esistere di quel pensiero.

A questo proposito, Merleau-Ponty non manca di enfatizzare che "il pensiero nel soggetto parlante non è una rappresentazione"54. Qui, il pensiero non è oggetto del soggetto parlante, non diventa oggettivazione da esso. Il "rappresentare" è il compito proprio della scienza, dove va inteso nel senso di mettere dinnanzi, avere di fronte e quindi poter districare, oggettivare. Nel saggio intitolato L'epoca delle immagini del mondo, Heidegger precisa che “'rappresentare' significa: condurre innanzi a sé e ricondurre a sé" 55 . La parola di colui che parla riproduce in me delle rappresentazioni? La comunicazione, cioè la comprensione della parola dell'altro, ovvero parola che anzitutto è gesto linguistico, non può essere frutto delle semplici rappresentazioni. Da questo punto di vista, si nota incisivamente il ruolo del corpo e del linguaggio in questa impresa intersoggettiva. La fenomenologia del linguaggio svolge, dunque, un ruolo essenziale di superare i dogmi della teoria della rappresentazione: "non ho bisogno di rappresentarmi la parola per saperla e per pronunciarla. Basta che ne possieda

\footnotetext{
52 MERLEAU-PONTY, M., Phénoménologie de la perception, 2004, p. 238. (Corsivo mio).

53 MERLEAU-PONTY, M., Phénoménologie de la perception, 2004, p. 218.

54 MERLEAU-PONTY, M., Phénoménologie de la perception, 2004, p. 219.

55 HEIDEGGER, M., Sentieri interrotti, 1997, p. 93.
} 
l'essenza articolare e sonora come una delle modulazioni, uno degli usi possibili del mio corpo" 56 .

Soltanto a titolo illustrativo, è rilevanti osservare in questo passo, ancora una volta, l'intreccio corpo e parola. In realtà, ciò che il filosofo vuole accennare è che "non è con delle 'rappresentazioni' o con un pensiero che io comunico per primo, ma con un soggetto parlante, con un certo stile d'essere e con il "mondo" a cui mira" 57 . In fondo, per uscire dall'impresa delle rappresentazioni, il soggetto parlante deve far manifestare, rivelare o incarnare la parola dal suo mondo linguistico nel momento stesso dell'atto di pronunciarla. Nella teoria della rappresentazione c'è sì un soggetto che parla, ma non è un soggetto parlante, è un soggetto pensante. Nel capitolo su Le cogito, Merleau-Ponty torna a ribadire, da un lato, che il parlare non è un'evocazione delle immagini verbali e, dall'altro, la parola non è una rappresentazione: "il soggetto parlante si getta nella parola senza rappresentarsi le parole che sta per pronunciare, la psicologia moderna elimina la parola come rappresentazione, come oggetto per la coscienza, e svela una presenza motoria della parola che non è la conoscenza della parola" ${ }^{8}$. Il linguaggio dunque è espressione e non segno, realizzazione del senso e non rappresentazione della coscienza.

Merleau-Ponty offre, inoltre, un'analisi ricca e dettagliata riguardo il rapporto fra la parola e il pensiero.

C'è quindi, sia in colui che ascolta, $[\ldots]$ sia in colui che parla, $[\ldots]$ un pensiero nella parola che l'intellettualismo non sospetta. [...] Anzitutto, la parola non è il "segno" del pensiero. [...] La parola e il pensiero [...] sono avvolti l'uno nell'altro, il senso è preso nella parola e la parola è l'esistenza esteriore del senso. Non potremo nemmeno ammettere, come si fa di solito, che la parola sia un semplice mezzo di fissazione, o anche l'involucro e il vestito del pensiero59.

La citazione che abbiamo appena proposto è di grande utilità poiché sintetizza mirabilmente due propositi del pensiero di Merleau-Ponty: il primo, la

${ }^{56}$ MERLEAU-PONTY, M., Phénoménologie de la perception, 2004, p. 220. (Corsivi miei).

57 MERLEAU-PONTY, M., Phénoménologie de la perception, 2004, p. 224.

$5^{8}$ MERLEAU-PONTY, M., Phénoménologie de la perception, 2004, p. 464.

59 MERLEAU-PONTY, M., Phénoménologie de la perception, 2004, p. 448. Nello scritto del '52, Un inédit, il filosofo ribadisce "dans ce domaine, il est plus aisé de montrer que le langage n'est jamais le simple vêtement d'une pensée qui se posséderait elle-même en toute clarté" (MERLEAUPONTY, M., Un inédit de Merleau-Ponty. Revue de Métaphysique et de Morale, 1962, p. 406). 
contrapposizione della teoria del segno e il secondo, invece, il rapporto chiastico d'enveloppement réciproque fra parola e pensiero. La prima parte della citazione inizia con una asserzione abbastanza stupefacente: "la parola non è il 'segno' del pensiero”. Anzitutto, il filosofo mette in discussione l'analisi dell'approccio linguistico di Saussure. Il senso viene posto come costituito nella comprensione come un concetto, e poi illustrato nel fenomeno dell'esperienza, in modo che l'esperienza consista piuttosto nella correzione dell'imperfezione del fenomeno in relazione al suo concetto. Dicendo che "il senso è preso nella parola", MerleauPonty indica la via da seguire: "essere preso" significa esattamente che, tra il fenomeno del segno e il fenomeno dell'esperienza che lo circonda, è rimasta solo un'esperienza e che la parola diventa in sé l'esperienza. Il gesto della parola rende questa parola presente a un'esteriorità e quindi si trasforma ad essa: "la parola è l'esistenza esteriore del senso", dice il filosofo.

La seconda parte tratta della figura chiastica d'enveloppement réciproque fra parola e pensiero: essi "sono avvolti l'uno nell'altro", afferma Merleau-Ponty. Si potrebbe suppore un rapporto chiastico. Tale citazione disegna perfettamente l'identità della futura ontologia charnelle, un pensiero ossessionato per la figura dell'enveloppant-enveloppé, perché il potere della carne è quello d'enveloppement, dell'atto fenomenico della reversibilità l'uno nell'altro, d'empiétement e dell'incrociarsi l'uno con l'altro. Secondo il filosofo, la parola deve cessare "d'essere una maniera di designare l'oggetto o il pensiero"6o. Le parole non possono essere più 'le 'fortezze del pensiero', [ma devono] diventare la presenza di questo pensiero nel mondo sensibile, e non il suo vestito, ma il suo emblema o il suo corpo" ${ }^{61}$. Questa citazione sembra essere il punto centrale della teoria del linguaggio di Merleau-Ponty. Riprendendo l'espressione "fortezze del pensiero", egli reagisce all'idea comune secondo cui la parola tende a mettere in una fortificazione il pensiero con l'intento di preservarlo dagli attacchi esterni. In realtà, le parole ci gettano nel cuore stesso delle parole dell'altro, ci aprono al mondo in cui s'espressa. Il passaggio al mondo e il passaggio al corpo non sono indifferenti per il senso perché è proprio il suo compito, cioè d'essere relazione ed ambiguità. Allora, il pensiero non viene prima della parola, ma piuttosto abita la parola, è la parola stessa messa in silenzio prima di apparire.

6o MERLEAU-PONTY, M., Phénoménologie de la perception, 2004, p. 222.

${ }_{61}$ MERLEAU-PONTY, M., Phénoménologie de la perception, 2004, p. 222. (Corsivi miei). 
Riprendendo ancora la stessa trattazione, l'autore propone di fare una netta distinzione fra il "designare", il "vestito" e l'“emblema”. Il "designare" è derivato da signum, segno. Il termine è impiegato come sinonimo di segnalare, indicare, significare. Designare è anche sinonimo di denotare; ad esempio, le parole bambino, piccino, fanciullo, hanno uguale denotazione perché designano lo stesso oggetto, anche se con differenti connotazioni. Il secondo termine da analizzare è quello del vestito. Esso è una specie di avvolto, di ammantato, di copertura. Vestirsi è coprire qualcuno con abiti, proteggere il corpo dal freddo, dallo sguardo dell'altro. L'emblema, invece, è derivato dal greco $\check{c} \mu \beta \lambda \eta \mu \alpha$, composto delle parole $\dot{\varepsilon} v$ e $\beta \dot{\alpha} \lambda \lambda \omega$, che significa letteralmente "cosa inserita" o "gettar dentro qualcosa". L'emblema è una figura simbolica e ha il potere di svelare ciò che è messo dentro, ad esempio, la bilancia è l'emblema della giustizia; lo scettro è emblema di sovranità. Riassumendo, la parola non è una maniera di disegnare l'oggetto o il pensiero né tantomeno il suo vestito. La parola quindi è l'emblema del pensiero, fa esistere il pensiero tramite il corpo e, allo stesso tempo, gli dà corpo, cioè consistenza ed esistenza. La parola è la deiscenza del pensiero. Quando parlo, non designo un senso, ma lo vivo e l'eseguo. In effetti, s’impara il linguaggio solo attraverso l'esistenza carnale.

Ma ciò che conviene qui esaminare è che la "parola" divenne il filo della texture charnelle fra il pensiero e il linguaggio. Il linguaggio è quindi condizione per il pensiero. Siccome il linguaggio è espressione corporea, così, in ultima analisi, il corpo è la condizione per il pensiero. La constatazione fatta da MerleauPonty sintetizza bene ciò che abbiamo appena delineato.

Il soggetto si pensa inseparabile dall'idea del corpo e dall'idea del mondo. [...] Il soggetto che io sono, concretamente preso, è inseparabile da questo corpo e da questo mondo. Il mondo e il corpo ontologici che ritroviamo nel cuore del soggetto non sono il mondo in idea o il corpo in idea, ma il mondo stesso contratto in una presa globale, è il corpo stesso come corpo-conoscente ${ }^{62}$.

Il linguaggio installa in noi l'apertura al dialogo con l'altro, ci ancre nel mondo culturale, sociale e linguistico. Merleau-Ponty mette qui in rilievo il carattere sociale del linguaggio. L'essenza del linguaggio e l’intenzione di parlare è

62 MERLEAU-PONTY, M., Phénoménologie de la perception, 2004, p. 469-470. 
quella "rivelazione dell'essere intimo"63 presente in ciascun soggetto parlante, dice il filosofo rifacendosi ad una citazione di Goldstein. L'atto del parlare stesso è il far incarnare la parola parlante nel mondo muto, anche se "noi viviamo in un mondo in cui la parola è instituita"64. Ogni volta l'atto del parlare è una texture charnelle di una storia di textures. Solo se abbiamo la capacità di parlare, possiamo costruire. Costruire è un far abitare il senso, è farlo engager. Il non-senso è quindi la non-abitazione. Il "senza senso" invece è il dis-abitato. Come abbiamo già visto, "il senso abita la parola"65 e che in esso l'espresso abita realmente e, non esiste, cioè senza l'espressione, e che colui che ascolta accoglie il pensiero della parola parlante. Infondo, per Merleau-Ponty la parola è capace di comunicare e condividere con gli altri il mondo attraverso il corpo. Ecco perché fra "tutte le operazioni espressive, la parola è unica capace di sedimentarsi e di costituire una acquisizione intersoggettiva" 66 . Il compito dei filosofi è pensare il non ancora pensato e dire il non ancora detto in un mondo già parlato. Ridire il dicibile ${ }^{67}$. Una cosa è veramente detta solo se non è completamente detta, è pensata solo se conserva un impensato. Il soggetto parlante abita mentre parla. La parola e il senso, sono sempre, secondo il loro diverso modo, indispensabile per l'abitare o per l'ancrer.

Tra pensiero e parola non c'è un rapporto estrinseco, bensì di attrazione intima e d'enveloppement réciproque evidente. Così come tra corpo e linguaggio, senso e parola, voce del silenzio e mondo, visibile e invisibile. Tutto lo sviluppo teorico che abbiamo appena evidenziato in questo item tende a mostrare che per Merleau-Ponty il pensiero non esiste fuori dalle parole, perché "la sua parola è il suo pensiero"68. In altri termini, la parola è l'atto con il quale il pensiero "si eternizza in verità"69. La parola non è la manifestazione di un pensiero già fatto, ma al contrario, la realizzazione del pensiero nell'atto di farsi e di disfarsi nella ed attraverso la parola. Un pensiero senza parola è assurdo come un'idea di una musica senza suoni. Nel saggio del '52 su Le lagage indirect et les voix du silence,

\footnotetext{
63 MERLEAU-PONTY, M., Phénoménologie de la perception, 2004, p. 238.

64 MERLEAU-PONTY, M., Phénoménologie de la perception, 2004, p. 224.

65 MERLEAU-PONTY, M., Phénoménologie de la perception, 2004, p. 234; 365.

66 MERLEAU-PONTY, M., Phénoménologie de la perception, 2004, p. 231.

67 Per una contestualizzazione della reversibilità merleau-pontyana di dicibile e indicibile, rimandiamo a CASTORIADIS, C., Le dicible et l'indicible. L'Arc, 1971, p. 67-79.

68 MERLEAU-PONTY, M., Phénoménologie de la perception, 2004, p. 219.

69 MERLEAU-PONTY, M., Phénoménologie de la perception, 2004, p. 448.
} 
il filosofo ribadisce: "il linguaggio significa quando, invece di copiare il pensiero, si lascia disfare e rifare da esso"70.

\section{La fenomenologia del gesto}

2.1 «La parola è un vero gesto»

Come pensare l'esperienza del gesto? Ma che cos'è il fenomeno gestuale in rapporto al fenomeno della parola? Il fenomeno del corpo ci ha rimandato a quello della parola e il problema della parola a quello del gesto. Passiamo ora a dare qualche precisazione riguardo al "ritorno" al fenomeno del gesto. Esso è complesso, perciò è assai importante fare una delimitazione delle nostre considerazioni. Non ci soffermeremo nel dibattito dell'inscrizione del gesto come movimento corporeo. Il gesto qui è inteso come una figura disegnata a partire dal movimento del corpo, per esempio, un movimento della mano nel momento di far un segno a un amico, un movimento del braccio o della testa71. Ciò che caratterizza questo gesto è la sua capacità di muoversi effettivamente e si tratta infatti di una motricità corporea. Tramite il corpo, la gestualità è quindi la prima naissance del movimento. Soffermiamoci soltanto di mettere in discussione il problema del fenomeno del gesto rispetto a quello della parola. Oltre questo, cercheremo brevemente di argomentare riguardo la possibilità o l'impossibilità dell'equivalenza del gesto e della parola, così come si approda la tesi evidenziata nella Phénoménologie.

Il tema merleau-pontyano del rapporto parola e gesto si sintetizza in questa espressione: "la parola è un vero gesto e contiene il suo senso come il gesto contiene il suo. È ciò che rende possibile la comunicazione" 72 . Prima di ogni altra definizione, il filosofo circoscrive che "la parola è un vero gesto". Poi aggiunge che la comunicazione umana è possibile solo come gesto corporeo. E ancora più determinante, alla fine del paragrafo egli ribadisce ancora: "la parola è un gesto e la sua significazione un mondo"73. Nel saggio del '51, Sur la phénoménologie du

\footnotetext{
70 MERLEAU-PONTY, M., Signes, 2001, p. 72.

${ }^{71}$ MERLEAU-PONTY, M., Phénoménologie de la perception, 2004, p. 141.

72 MERLEAU-PONTY, M., Phénoménologie de la perception, 2004, p. 224.

73 MERLEAU-PONTY, M., Phénoménologie de la perception, 2004, p. 224. È inevitabile che qui una certa domanda venga posta. Di quale mondo si tratta? È sicuramente il mondo anteriore all'oggettività e alla scienza, è il mondo selvaggio e grezzo. Secondo Merleau-Ponty, è quel mondo inteso "comme berceau des significations, sens de tous les sens, et sol de toutes les pensées. [...] Ce
} 
langage, ripreso in Signes, Merleau-Ponty afferma: "se la parola è paragonabile a un gesto, ciò che essa viene caricata di esprimere sarà con essa nello stesso rapporto dell'obiettivo con il gesto che lo mira" 74 . Da queste concezioni, la parola va ricollocata pari pari nella dinamicità fenomenologica del gesto. In fondo, il problema del gesto e della parola sono strettamente legati. Come può la parola essere gesto? È della sua propria natura essere gestuale. Per rispondere a questa domanda si rivela necessario ricollocare la parola all'interno di quella texture di gestualità e di prassi in cui originariamente si trova. In effetti, è necessario ridare la parola al gesto, al corpo e allo stesso tempo il gesto alla parola. Ridare la parola al corpo significa far un ritorno alla percezione e al gesto stesso. Si cerchiamo di snodare meglio questa citazione, sembra che c'è una differenza tra il senso della parola e quello del gesto. Per Merleau-Ponty, la parola è "un segno convenzionale", il gesto invece è "un segno naturale".

Si vede bene cosa c'è di comune fra il gesto e il suo senso, per esempio fra l'espressione delle emozioni e le emozioni stesse: il sorriso, il volto disteso, l'allegria dei gesti contengono realmente il ritmo d'azione, il modo d'essere al mondo, che sono la gioia stessa. [...] È quanto si esprime di solito dicendo che il gesto o la mimica emozionale sono "segni naturali", la parola un "segno convenzionale". Ma le convenzioni sono un modo tardivo di relazione fra gli uomini, presuppongono una comunicazione preliminare ${ }^{75}$.

La citazione che abbiamo appena proposto è essenziale per comprendere il legame (lien) inscindibile fra il gesto e il suo senso. Per quanto riguarda la gesticolazione emozionale, si potrebbe dire che il gesto della carezza esprime l'amore? Carezza ed amore sono legati? Soltanto a titolo illustrativo, cerchiamo di esemplificare tale legame ricorrendo all'esempio della collera usato da MerleauPonty. Il gesto di rabbia, di furia e di ira espressa la collera? Allora, si percepisce la collera attraverso il suo gesto? Per comprendere il senso dei gesti di cui siamo noi stessi testimoni, visto che siamo noi a provarli, Merleau-Ponty ammette che non c'è bisogno di "ricordare i sentimenti che ho provato quando eseguivo per conto

n'est plus le déploiement visible d'une Pensée constituante, ni un assemblage fortuit de parties, ni, bien entendu, l'opération d'une Pensée directrice sur une matière indifférente, mais la patrie de toute rationalité" (MERLEAU-PONTY, M., Phénoménologie de la perception, 2004, p. 493).

74 MERLEAU-PONTY, M., Signes, 2001, p. 145.

75 MERLEAU-PONTY, M., Phénoménologie de la perception, 2004, p. 227. 
mio i medesimi gesti” ${ }^{6}$. Allora, come si conosce la mimica della collera? La si conosce dall'interno? Si la percepisce come un fatto psichico nascosto dietro il suo gesto? La collera la si legge "nel gesto, il gesto non mi fa pensare alla collera, ma è la collera stessa" 77 . L'altra considerazione fatta da Merleau-Ponty è che la nostra comprensione dei gesti sarebbe per lo più limitata ai gesti umani, visto che non si comprende affatto la mimica sessuale del "maggiolino o della mantide religiosa" 78 .

Il senso dei gesti viene compreso e afferrato tramite un atto dallo spettatore. Tale comprensione dei gesti è ottenuta grazie alla

reciprocità delle mie intenzioni e dei gesti altrui, dei miei gesti e delle intenzioni leggibili nella condotta altrui. Tutto avviene come se l'intenzione dell'altro abitasse il mio corpo o come se le mie intenzioni abitassero il suo. Il gesto di cui sono testimone disegna a linee tratteggiate un oggetto intenzionale ${ }^{79}$.

Quando si fa un gesto a un amico per dirgli di avvicinarsi oppure soltanto per salutarlo, visto la distanza che ci separa da lui, ad ogni momento del movimento gestuale, proviamo l'esistenza di un'intenzione come parte presente e reale del corpo vivente. Merleau-Ponty evidenzia che la mia intenzione s'esprime a distanza attraverso il gesto tramite il corpo. Così, a dire il vero, "il corpo si fa gesto" ${ }^{\circ}$. Il gesto, di per sé, è un'espressione. In questa prospettiva, qui si trova la reciprocità delle mie intenzioni e dei gesti altrui. La comprensione del senso del gesto della chiamata o del saluto non è un atto di interpretazione intellettuale, ma attraverso il mio corpo engagé al mondo, riconosco "come irriducibile il movimento con il quale io mi presto allo spettacolo, mi unisco ad esso in una specie di riconoscimento cieco che precede la definizione e l'elaborazione intellettuale del senso"81. In sintesi, il senso del gesto non è né davanti né dietro né al di là di esso, ma si può dire che "esso si diffonde sul gesto stesso; [il gesto] disegna da sé stesso il suo senso" 82 .

La citazione che abbiamo evidenziata parla del gesto di cui sono testimone e dell'oggetto intenzionale. Quale sarebbe il presunto gesto? Qual è l'oggetto

76 MERLEAU-PONTY, M., Phénoménologie de la perception, 2004, p. 225.

77 MERLEAU-PONTY, M., Phénoménologie de la perception, 2004, p. 225.

78 MERLEAU-PONTY, M., Phénoménologie de la perception, 2004, p. 225.

79 MERLEAU-PONTY, M., Phénoménologie de la perception, 2004, p. 225.

80 MERLEAU-PONTY, M., Signes, 2001, p. 392.

81 MERLEAU-PONTY, M., Phénoménologie de la perception, 2004, p. 226.

82 MERLEAU-PONTY, M., Phénoménologie de la perception, 2004, p. 226. 
intenzionale? Proseguendo l'analisi, si potrebbe dedurre che il movimento corporale per farsi vedere all'amico d'avvicinarsi o per salutarlo è di per sé il gesto. L'intenzione, invece, significa il mirare a, il tendere a, vale a dire l'intento di salutare l'amico distante. Questo "mirare a" che l'intenzionalità designa è l'intento di qualche cosa che non è ancora presente, ma che si vuole raggiungere. In quest'ottica, l’intenzionalità non significa la pura coscienza di qualche cosa, ma quella che mira verso il mondo in cui si vive. L'intenzionalità, infatti, è l'atto di rivelare il senso del gesto presente nell'essere della carne del mondo, è quell'atto di significare, far il senso sfuggire da se stesso verso un altro. Da questo punto di vista, l'intenzione può essere effettivamente il punto di passaggio dal mio movimento gestuale-carnale verso il mondo sensibile-selvaggio. Il gesto divenne l'entre-deux o il framezzo tra il testimone, colui che ha il movimento gestuale e quello dell'oggetto intenzionale, cioè l'amico a cui dal testimone si dirige il gesto. Riassumendo, il gesto che si fa a un amico è la prima bozza della significazione da creare, perché si scopre tutta la possibilità della esistenza. È in quest'ottica che Merleau-Ponty afferma che la parola sia più un gesto che un sistema di concetti ${ }^{83}$.

\subsection{Il gesto come fenomeno d"indicazione" nel mondo sensibile}

Torniamo dunque al discorso del legame fra il gesto e il suo senso. Per documentare questo, soffermiamoci a partire dal punto di vista della cultura. L'intento nostro non è di fare né un discorso naturalistico né culturalistico, ma rilevare l'aspetto incarnato del gesto e del suo senso rispetto all'organizzazione corporea e del modo stesso di accogliere la situazione e di viverla 84 nella diversità culturale. È necessario, dunque, introdurci o scivolarsi nel modo di esistere di quell'ambiente gestuale per poter comprendere il senso dei gesti di quella specifica cultura.

${ }_{33}$ Cf. SU, H.-C., La notion de geste chez Merleau-Ponty, 2018, p. 30.

${ }^{8}$ Cf. MERLEAU-PONTY, M., Phénoménologie de la perception, 2004, p. 230. In una nota di lavoro del maggio del '6o ne Le Visible et l'Invisible, Merleau-Ponty ribadisce, ancora più radicalmente, questa posizione: "la distinction des 2 plans (naturel et culturel) est d'ailleurs abstraite: tout est culturel en nous (notre Lebenswelt est 'subjectif') (notre perception est culturelle-historique) et tout est naturel en nous (même le culturel repose sur le polymorphisme de l'Être sauvage)" (MERLEAU-PONTY, M., Le visible et l'invisible, 1979, p. 301). I gesti del nostro essere tramite il corpo sono perciò al tempo stesso naturali e culturali, vale a dire come il diritto e il rovescio di un medesimo foglio. Sostiene ancora il filosofo: "il n'est pas plus naturel ou pas moins conventionnel de crier dans la colère ou d'embrasser dans l'amour que d'appeler table une table" (MERLEAU-PONTY, M., Phénoménologie de la perception, 2004, p. 230). 
Merleau-Ponty riesce raggiungere congiuntamente o reciprocamente il senso della parola e quello del gesto. Per chiarire questo rapporto, il filosofo evidenzia che

è pur necessario che qui il senso delle parole sia finalmente indotto dalle parole stesse, o più esattamente, che il loro significato concettuale si formi per prelevamento su un significato gestuale, che, esso, è immanente alla parola. E come, in un paese straniero, comincio a comprendere il senso delle parole dal loro posto in un contesto d'azione e partecipando alla vita comune - coì come un testo filosofico ancora mal compreso mi rivela per lo meno un certo "stile" - sia uno stile spinoziano, criticistico o fenomenologico - che è il primo abbozzo del suo senso, comincio a comprendere una filosofia scivolandomi nel modo di esistere di questo pensiero ${ }^{85}$.

Come si può intuire, il "significato gestuale" di cui Merleau-Ponty parla riguarda l'azione corporea, vale a dire l'espressione corporea. La base corporea rivela l'apertura alla partecipazione alla vita comune di una comunità coesistenziale. Per il filosofo, in un paese straniero, si comprende il senso delle parole solo dal loro posto. Il "posto" significa il campo d'azione e di partecipazione, quel posto allo spazio e al tempo, cioè il campo d'apertura all'essere. L'azione, invece, richiama sempre l'espressione corporea altrimenti non ci sarebbe movimento gestuale-carnale. La "partecipazione" alla loro vita sociale riguarda l'evidenza del fenomeno dell'engagement del corpo a quella specifica cultura linguistica e gestuale. La parola dunque si realizza secondo il gesto corporeo. Il corpo e la parola si uniscono nel fenomeno del linguaggio. Tale fenomeno è possibile grazie lo scivolarsi del corpo e della parola vicendevolmente nella texture della chair del linguaggio. Il glisser (scivolare) è un termine chiave nell'ontologico merleau-pontyana.

Ora, bisogna innanzitutto notare che, l'engagement al mondo attraverso il corpo e l'atto di glisser sono legati ed intrecciati nell'ontologia della chair, dato che senza l'engagement alla chair du monde non esisterebbe neanche il fenomeno di glissement ad esso. Scivolare significa inserirsi o introdursi abilmente e delicatamente nella stretta fessura dell'essere. L'essere di per sé è scivoloso. Io m'engage, quindi scivolo. Nella teoria della chair del linguaggio, parlare è un atto di glissement nella texture charnelle delle parole. Potremmo inoltre evidenziare che il glisser è anche sinonimo di sfuggimento. Come già si è affermato, grazie a

85 MERLEAU-PONTY, M., Phénoménologie de la perception, 2004, p. 219. 
questa specie di sfuggimento, nell'uomo è impossibile sostenere la sovranità di uno strato di comportamenti chiamato naturali o gestuale su un mondo culturale chiamato linguistico o viceversa. Ecco perché il fenomeno di sfuggimento serve a definire l'uomo come sfuggente a sé nel suo proiettarsi nel cuore stesso dell'essere. Lo sfuggente è proprio superamento verso ciò che gli sfugge, si supera verso l'essere. La realtà umana per cui lo sfuggimento appare al mondo deve essere esso stesso uno sfuggimento continuo. Lo sfuggimento, quindi, non è l'atto di scappare inavvertitamente, bensì l'atto di glisser nel linguaggio dell'essere. Se lo sfuggimento tende verso l'essere, è perché, con il suo scivolarsi va verso l'essere che è solo fondamento del suo essere sfuggente, qualificandosi nel suo essere come l'essere nella chair $d u$ monde. Il soggetto parlante è scivoloso, inafferrabile, ambiguo. Sia il modo di esistere di questo sfuggimento come del glissement, entrambi si "suppone che sia già nel mondo o nell'essere" 86.

Da quanto sottolineato sinora, Merleau-Ponty vede il gesto nella parola. Essa ha un senso come il gesto ha il suo. Nel pensiero merleau-pontyano il "senso" non opera né meccanicamente né foneticamente. Da questa indagine si può dedurre un fatto ultimo, cioè quello del "potere aperto e indefinito di significare" 87 . Il potere di "significare" è una questione della fenomenologia. Il gesto ha di per sé il potere di significare. Com'è noto, "il proprio del gesto umano è di significare al di là dalla sua semplice esistenza di fatto, d'inaugurare un senso" $88 \mathrm{e}$, perciò, da questo risulta che ogni gesto annuncia sempre un inizio e una continuazione. Questa esistenza aperta fa del senso della parola e del gesto acquisire un vero valore espressivo il cui significato non sarà mai esaurito.

L'ultimo aspetto da considerare riguardo il gesto è quello del suo potere d'“indicare" in senso fenomenologico. Che cosa il gesto indica? Prendiamo, allora, due citazioni di Le corps comme expression et la parole per illustrare la concezione data da Merleau-Ponty. La prima, il filosofo afferma che "il gesto [...] mi indica certi punti sensibili del mondo, mi invita a raggiungerlo"89. La parola,

\footnotetext{
86 MERLEAU-PONTY, M., Le visible et l'invisible, 1979, p. 290. Al riguardo, Barbaras riassume bene tale rapporto e commenta che il significato linguistico si estende e realizza il significato gestuale mostrato dai comportamenti muti. In fondo, sia il significato gestuale come il linguistico, entrambi sono sempre connessi con il modo di esistenza. Perciò, si può riconoscere un significato gestuale o esistenziale della parola così come un significato concettuale o esistenziale del gesto. Cf. BARBARAS, R., Le tournant de l'expérience, 2013, p. 189.

87 MERLEAU-PONTY, M., Phénoménologie de la perception, 2004, p. 236.

88 MERLEAU-PONTY, M., Signes, 2001, p. 109.

89 MERLEAU-PONTY, M., Phénoménologie de la perception, 2004, p. 225. (Corsivi miei).
} 
come sottolinea Merleau-Ponty, non è soltanto gesto, ma è gesto che si fa indicazione. L'indicare significa rivelare, mostrare, manifestare, esprimere oppure svelare oltre. L'indicare è proprio della fenomenologia come lo è anche il significare e l'apparire. Si può ulteriormente evidenziare tali esempi: il corpo indica engagement al mondo e al linguaggio; l'incarnazione indica l'essere incarnato nella chair du monde; il dolore indica la malattia. Il primo aspetto che si deve sottolineare da questa citazione è che il gesto indica il mondo aprendosi e scivolandosi ad esso. Il gesto mostra con un cenno le realtà fenomeniche del mondo circonstante. Ogni gesto è chair du monde, altrimenti non sarebbe ciò che è, cioè gesto visibile dall'invisibile. Solo chi appartiene al mondo può indicare. L'indicare è proprio di chi appartiene alla chair du monde. Il gesto ha la meta d'indicare i punti sensibili del mondo, la tutta realtà percettiva che è attorno a noi, dato che siamo enveloppant-enveloppé di questa realtà sensibile, cioè di questa "carne del sensibile" 90 . Questo sensibile, come già si è affermato "è la carne del mondo" 91. Detto tutto questo, vale ricordare che l'indicare nel senso fenomenologico non "indica" soltanto, ma invita. Invitare significa partecipare, inerire, raggiungere. Da questo punto di vista, oltre il livello fenomenologico del gesto in quanto indicazione, si svela anche il suo livello ontologico. Ora, nel gesto è implicito l'invito a pervenire ad approdare al mondo. L'espressione merleaupontyana "mi invita a raggiungerlo" non è altro che il senso dell'engager ontologico "del mio mondo come carnale e del mondo d'altri" 92.

La seconda citazione, invece, sembra quasi simile à quella di prima: "il gesto si limita ad indicare un certo rapporto fra l'uomo e il mondo sensibile"93. Il gesto è questo fenomeno d'entre-deux fra l'uomo e il mondo. È ciò che rende anche possibile tale rapporto. In questo caso, dunque, il corpo si fa gesto perché è un corpo engagé, è una chair du corps, è un corpo che si fa mostrare. Nel saggio su Le lagage indirect et les voix du silence, il filosofo riafferma con chiarezza che "il nostro corpo, mentre vive e si fa gesto, s'affida solo sul suo sforzo per essere al mondo, si tiene in piedi perché la sua inclinazione è verso l'alto, perché i suoi campi percettivi lo spingono verso questa posizione rischiosa" 94 . La citazione che abbiamo appena proposto è di grande utilità poiché sintetizza mirabilmente la

90 MERLEAU-PONTY, M., Signes, 2001, p. 272. (Corsivi miei).

91 MERLEAU-PONTY, M., La nature, 1995, p. 280.

92 MERLEAU-PONTY, M., La nature, 1995, p. 287.

93 MERLEAU-PONTY, M., Phénoménologie de la perception, 2004, p. 227. (Corsivo mio).

94 MERLEAU-PONTY, M., Signes, 2001, p. 111. 
visione merleau-pontyana dell'equivalenza del gesto e della parola. La portata teorica è assai evidente, visto che l'autore riporta la chair du corps in cui si può trovare il lien fra gesto e parola. È quindi attraverso il corpo chair che si intelaia il significato linguistico e quello gestuale. Con una precisazione teoretica del legame del fenomeno del corpo e della parola, Merleau-Ponty riassume dicendo che "io mi rapporto alla parola come la mia mano si dirige verso il luogo del mio corpo che viene punto"95. Rilevanti sono le considerazioni di Su al riguardo: "fare un gesto è rompere il silenzio in cui viviamo e far apparire la sua esistenza carnale al mondo. Nel seno del mondo, il mio gesto avvolge le cose e le cose avvolgono il mio gesto" ${ }^{96}$.

\section{Considerazioni finali}

"Ritornare" al fenomeno della parola e del gesto. Ecco l'obiettivo sollevato in questo articolo. Merleau-Ponty alza la propria voce in difesa della parola. La tutela della parola coincide con la tutela dell'uomo (soggetto parlante). La parola si fa gesto attraverso il corpo. Esso è gestualità per eccellenza grazie al suo engagement al mondo. Non c'è soltanto soggetto pensante, ma soggetto parlante. "Ritorno": questo è il termine giusto per celebrare il $75^{\text {mo }}$ anniversario della comparsa della Phénoménologie de la perception. "Ritornare" per rifare la texture rotta a causa della corruzione della parola e della perdita del contatto con la realtà. Senza contatto con essa ci si abitua a confondere la realtà autentica (verità) di quella manipolata per i produttori e diffusori di abusi di parole: i fake-news.

Ciò che risulta fondamentale pensare è il fatto che il fenomeno della parola e del gesto sono collegati a quello della nozione tardiva della chair. Nel seno di una comunicazione muta del mio gesto a distanza, questo atto gestuale di trascendenza rivela il rapporto che mi collega (lien) silenziosamente alle cose ed ad altri tramite la texture charnelle. Nel senso del gesto c'è sempre qualcosa che non è ancora completamente dato o rivelato e, perciò, questo invisibile è intrecciato con il dato visibile e, quindi, è in filigrana attraverso di esso. Di questo presupposto, si vede che Merleau-Ponty mira una teoria della chair del linguaggio. Il linguaggio, come abbiamo dimostrato, è anzitutto articolazione dell'essere gestuale.

\footnotetext{
95 MERLEAU-PONTY, M., Phénoménologie de la perception, 2004, p. 220.
}

96 SU, H.-C., La notion de geste chez Merleau-Ponty, 2018, p. 58. 
Fare un gesto è riprendere il dato sensibile del mondo e, allo stesso tempo, dare al mondo una nuova espressione che è la verità. È fare in modo tale che il mondo s'organizzi avvolto a me, come se le cose diventassero i miei gesti e i miei gesti delle cose. In sintesi, il gesto è un toucher a distanza la chair du corps e la chair du monde.

\section{Bibliografia}

BARBARAS, R., Le tournant de l'expérience: recherches sur la philosophie de Merleau-Ponty. Paris: Vrin, 2013.

CASTORIADIS, C., Le dicible et l'indicible. Hommage à Merleau-Ponty. L'Arc, Aix-en-Provence, n. 46, p. 67-69, 1971.

FANIZZA, F., Motivi estetici nella fenomenologia di Merleau-Ponty. Aut Aut, Milano, v. 66, p. 516-519, 1961.

GAMBAZZI, P., La piega e il pensiero. Sull'ontologia di Merleau-Ponty. Aut Aut, Milano, n. 262/263, p. 21-47, 1994.

HEIDEGGER, M., Sentieri interrotti. Firenze: La nuova Italia, 1997.

LAWLOR, L., Le chiasme et le pli. Une introduction au concept philosophique d'archéologie. In: CARIOU, M. (org). Merleau-Ponty aux frontières de l'invisible. Milano: Mimesis, 2003, 191-203.

MERLEAU-PONTY, M., Phénoménologie de la perception. Paris: Gallimard, 2004 .

MERLEAU-PONTY, M.Éloge de la philosophie et autres essais. Paris: Gallimard, 1960 .

MERLEAU-PONTY, M. Sens et non-sens, Paris 1948.

MERLEAU-PONTY, M. Signes. Paris: Gallimard, 2001.

MERLEAU-PONTY, M. Un inédit de Merleau-Ponty. Revue de Métaphysique et de Morale, Paris, v. 4, p. 401-409, 1962.

MERLEAU-PONTY, M. L'œil et l'esprit. Paris: Gallimard, 2007.

MERLEAU-PONTY, M. Le visible et l’invisible. Paris: Gallimard, 1979.

MERLEAU-PONTY, M. Résumés de cours. Collège de France 1952-196o. Paris: Gallimard, 1968.

MERLEAU-PONTY, M. La prose du monde. Paris: Gallimard, 1992. 
MERLEAU-PONTY, M. La Nature. Notes de cours du Collège de France. Paris: Seuil, 1995.

MERLEAU-PONTY, M. Notes des cours 1958-59 et 1960-61. Paris: Gallimard, 1996.

MERLEAU-PONTY, M. Notes de travail inédites de 1958, 1959 et 196o, Bibliothèque Nationale de France (B.N.), volume VIII, 2.

MERLEAU-PONTY, M. Parcours II 1951-1961. Lagrasse: Verdier, 2000.

NOBLE, S. A., "Entre le silence des choses et la parole philosophique”. MerleauPonty, Fink et les paradoxes du langage. Chiasmi International, Milano, v. 6, p. 111-143, 2005.

SEGGIARO, N., La chair et le pli. Merleau-Ponty, Deleuze e la multivocità dell'essere. Milano: Mimesis, 2009.

SU, H.-C., La notion de geste chez Merleau-Ponty: corp, langage et silence. Beau Bassin, 2018.

THIERRY, Y., Du corps parlant: le langage chez Merleau-Ponty. Paris: Ousia, 1987.

WALDENFELS, B., Vérité à faire: la questione della verità in Merleau-Ponty. In: BOETTI, A.-M. S., La prosa del mondo: omaggio a Maurice Merleau-Ponty. Napoli: QuattroVenti, 1990.

Recebido em: 26/12/2019. Aprovado em: 17/03/2020. Publicado em: 18/07/2020. 